(NASA-CR-199997) TWO-STREAM MODELING CF PLASMASPHERIC REFILLING

(NASA. Marshall space Flight

Center) $8 p$
N96-19523

Unclas

G3/75 0101211 


\title{
Two-stream modeling of plasmaspheric refilling
}

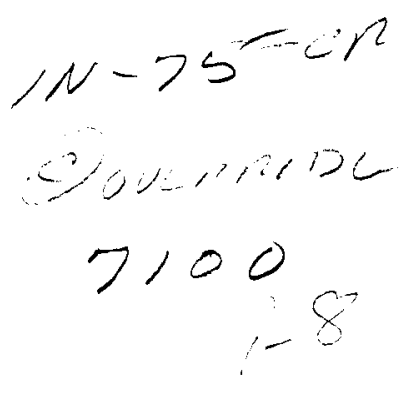

T. I. Gombosi and C. E. Rasmussen

Space Physics Research Laboratory, University of Michigan, Ann Arbor

\begin{abstract}
Plasmaspheric refilling on an $L=4$ flux tube was studied by using a time-dependent, hydrodynamic plasmaspheric flow model in which the ion streams from the two hemispheres are treated as distinct fluids. In the model the continuity, momentum, and energy equations of a twoion $\left(\mathrm{O}^{+}\right.$and $\left.\mathrm{H}^{+}\right)$, quasi-neutral, currentless plasma are solved along a closed geomagnetic field line; diffusive equilibrium is not assumed. Collisions between all stream pairs and with neutral species are included. The model includes a corotating, tilted dipole magnetic field and neutral winds. Ionospheric sources and sinks are accounted for in a self-consistent manner. Electrons are assumed to be heated by photoelectrons. The model flux tube extends from $200-\mathrm{km}$ altitude in one hemisphere to $200-\mathrm{km}$ altitude in the other hemisphere. Initially, the upwelling streams pass through each other practically unimpeded. When the streams approach the boundary in the conjugate ionosphere, a shock develops there, which moves upward and dissipates slowly; at about the same time a reverse shock develops in the hemisphere of origin, which moves upward. After about 1 hour, large shocks develop in each stream near the equator; these shocks move toward the equator and downward after crossing the equator. However, these shocks are probably artificial, because counterstreaming flows occur in each $\mathrm{H}^{+}$fluid, which the model can only handle by creating shocks.
\end{abstract}

\section{Introduction}

A long-standing question in plasmaspheric refilling has been whether the ion streams that come from each hemisphere would form an electrostatic shock pair when they meet at the equator or whether they would pass through each other [Banks et al., 1971; Schulz and Koons, 1972]. Even if the streams pass through each other, it is possible that they could excite waves that would change the pitch angle of the ions, leading to ion trapping [Schulz and Koons, 1972]. In order to model this, however, it is necessary to have a model in which the ion streams from conjugate hemispheres are distinguished; this is called a two-stream model.

Previous work involving two-stream models has been described by Singh [1988], Rasmussen and Schunk [1988], Singh and Torr [1990], Singh [1991], and Singh and Chan [1992]. Singh [1988] applied a two-stream version of the model described by Singh et al. [1986] to refilling on an $L=6$ field line. In this model, only the continuity and momentum equations were solved; the ion and electron temperatures were assumed to be constant. In addition, a Boltzmann relation was assumed to hold between electron density and electric potential:

$$
n_{e}=n_{o} \exp \left(\frac{\phi}{k T_{e}}\right)
$$

Copyright 1995 by the American Geophysical Union.

Paper number 95JA00081.

0148-0227/95/95JA-00081\$05.00 where $n_{e}$ is the electron density, $n_{o}$ is the electron density at a reference altitude, $\phi$ is the electric potential, which is assumed to be zero at the reference altitude, and $T_{e}$ is the electron temperature. This relation is used to calculate the electric field. In this study it was assumed that $T_{e}=10 T_{i}$ in order to reduce computer time. Singh [1988] found that the plasma streams pass through each other without being seriously affected by the other stream. Rasmussen and Schunk [1988] also developed a two-stream version of the Singh et al. [1986] model and applied it to refilling on an $L=4$ field line, but with $T_{e}=T_{i}$. Results were given both with and without $\mathrm{H}^{+}-\mathrm{H}^{+}$collisions. There was not much difference between these two cases, but the streams were reflected at higher altitudes when collisions were included. Rasmussen and Schunk [1988] find that the streams initially interpenetrate. The density fronts steepen into shocks as they travel toward the conjugate ionosphere; these shocks pass through the boundaries. Thereafter, reverse shocks develop in the hemisphere of origin for each stream, travel toward the conjugate hemisphere, and reverse direction. The subsequent motions of the shocks give results similar to those of one-stream models, with refilling between the shocks. They conclude that refilling eventually occurs from the equator downward.

Singh and Torr [1990] described a two-stresin model in which the temperatures are allowed to be anisotropic. In this model the ion continuity, momentum, and paralle: and perpendicular energy equations were solved. However, production and loss processes, heat flow, and collisions were neglected. In addition, a Boltzmann relation was assumed to hold between electron density and electric potential and was used to calculate the electric field. The electron temperature was assumed to be constant. Singh and Torr [1990] used this model to study 
plasmaspheric refilling in the presence of equatorial transverse ion heating, on an $L=4$ field line. This transverse heating represented the effect of wave-particle interactions. The transverse heating results in a temperature anisotropy with $T_{\perp}>T_{\|}$, which can stop the interhemispheric plasma flow for even moderate heating levels. This launches a shock wave, which propagates from the equator to the ionosphere from which the flow originated. Thereafter a quasi-steady state prevails. $T_{\perp}$ is greater than $T_{\|}$within 15 degrees of the magnetic equator, but at higher latitudes the anisotropy is reversed. The density profile has a broad maximum near the equator and minima at midlatitudes. There is also a local minimum right at the equator. Singh and Torr [1990] claim that the equatorial density maximum is analogous to the observations of trapped ions from DE 1 [Olsen, 1981; Olsen et al., 1987]. However, it is inconsistent to include large heating levels without allowing for the possibility of heat flow; Singh and Chan [1992] argue that the classical form of heat flow is not valid, since the mean free path is much greater than the scale length for temperature variations.

Singh [1991] compared results for plasmaspheric refilling found by using a two-stream model with anisotropic temperatures to those found when the temperatures are isotropic. In this work, Coulomb collisions were included, and there was no equatorial heating. The results are similar in an initial period ( $<5$ hours), characterized by supersonic flows from the ionospheres and the formation of shocks after the plasma streams pass the equator; these shocks move back toward the equator and then down to the ionospheres. Thereafter a temperature anisotropy develops with $T_{\|}>T_{1}$, causing a downward force, which prevents plasma behind a shock from following it as it crosses the equator. After the shock crosses the equator, the enhancement in $T_{\|}$is much greater than that in $T_{\perp}$, with the result that the downward pressure gradient force exceeds the upward mirror force, accelerating the shock downward. This shock moves down much faster than in the isotropic case. Due to the temperature anisotropy, the flux tube remains depleted until Coulomb collisions allow the anisotropy to relax. This begins at 7.68 hours, when the anisotropy relaxes near the ionospheric boundaries, allowing upward subsonic flow from the ionosphere. Singh [1991] found that there was minimal refilling during the initial period of supersonic flow; the majority of the refilling occurs in the following subsonic stage. The subsonic flows were found to last for several days.

Singh and Chan [1992] studied plasmaspheric refilling on an $L=4$ field line in the presence of equatorial transverse heating with a model that included Coulomb collisions. The early time results were not significantly different from those shown by Singh and Torr [1990]. At later times, Coulomb collisions cause the temperature anisotropy to relax, and upward subsonic flows develop, similar to what was shown by Singh [1991]. Singh and Chan [1992] also give results from a kinetic simulation that shows that the electrostatic shocks form at the mirror points of the equatorially heated ions.

In this paper, results will be presented for plasmaspheric refilling on an $L=4$ field line; these results were found by using a two-stream version of the plasmaspheric flow model, which we developed [Guiter et al., 1991]. This is a time-dependent hydrodynamic model in which the $\mathrm{H}^{+}$and $\mathrm{O}^{+}$continuity, momentum, and energy equations and the electron energy equation are solved along a closed dipole field line. The temperatures are assumed to be isotropic. The model includes the effects of ionization, charge exchange, recombination, collisions, and heat conduction and allows for external heat sources. Collisions with neutral species are included in addition to Coulomb collisions. Production and loss processes and heat flows are included, in contrast to the model used by Singh and coworkers [Singh and Torr, 1990; Singh, 1991; Singh and Chan, 1992]. Photoelectron heating of thermal electrons and a neutral wind profile are assumed. Although neutral winds do not directly affect plasmaspheric density, they do affect the ionospheric density profile and thus indirectly the plasmaspheric density. The ends of the model flux tube are at $200-\mathrm{km}$ altitude, which is low enough so that realistic inflow boundary conditions can be used. For this work the initial density profile was similar to that used by Rasmussen and Schunk [1988]. Improvements over previous studies include realistic ionospheric boundary conditions and the influence of $\mathrm{O}^{+}$streams (primarily as a source for $\mathrm{H}^{+}$) on plasmaspheric refilling. Results are presented from only the first hour of the calculation, because artificial shocks develop in each $\mathrm{H}^{+}$fluid by that time, due to the presence of counterstreaming flows within each fluid. However, interesting features develop in the ionosphcric region, which has not been included in previous two-stream refilling studies.

\section{Model}

For this work the streams of both $\mathrm{H}^{+}$and $\mathrm{O}^{+}$from the conjugate ionospheres were treated as distinct fluids, with all flows assumed to be field aligned. Ions that originate from the conjugate hemisphere are called outflowing (out of the plasmasphere and into the neighboring ionosphere), whereas ions that originate in the given hemisphere are called inflowing (into the plasmasphere from the neighboring ionosphere). The timedependent continuity, momentum, and energy equations for both streams of $\mathrm{O}^{+}$and $\mathrm{H}^{+}$ions were solved along a closed dipolar field line. However, the electron gas was treated as a one-stream fluid. Electron number density and velocity were found by assuming the plasma to be quasi-neutral and currentless. The electron energy equation was solved in order to obtain the electron temperature. These equations were presented by Guiter and Gombosi [1990].

In this model the collision terms were assumed to have the following form:

$$
\begin{gathered}
S_{s}=m_{s}\left(v_{s o} n_{o}-L_{s} n_{s}\right) \\
\frac{\delta M_{s}}{\delta t}=\sum_{t} n_{s} m_{s} v_{s t}\left(u_{t}-u_{s}\right) \Phi_{s t}+m_{s} v_{s o} n_{o}\left(u_{o}-u_{s}\right) \\
\frac{\delta E_{s}}{\delta t}=\sum_{t} \frac{n_{s} m_{s} v_{s t}}{m_{s}+m_{t}}\left[3 k\left(T_{t}-T_{s}\right) \Psi_{s t}+m_{t}\left(u_{t}-u_{s}\right)^{2} \Phi_{s t}\right] \\
+m_{s} v_{s o} n_{o}\left[\frac{3 k T_{o}}{2 m_{s}}+\frac{1}{2}\left(u_{o}-u_{s}\right)^{2}\right]-\frac{3}{2} L_{s} n_{s} k T_{s}
\end{gathered}
$$

In these equations the summations run over all species, including the neutral species; $v_{s t}$ is the momentum transfer collision frequency of species $s$ with species $t, v_{s o}$ is the production frequency of species $s$, and $L_{s}$ is the loss frequency of species $s$; $n_{o}, u_{o}$, and $T_{o}$ refer to the density, field-aligned velocity, and temperature of the parent neutral species. The velocitydependent correction factors $\Psi_{s t}$ and $\Phi_{s t}$ [cf. Tanenbaum, 1967; Burgers, 1969; Schunk, 1977] were calculated for the interaction of a given $\mathrm{H}^{+}$stream with other ion streams and with 
neutral species. Collisions between all stream pairs and with neutral species were included. Collision frequencies for $\mathrm{H}^{+}-\mathrm{H}^{+}$ and $\mathrm{O}^{+}-\mathrm{O}^{+}$interactions were taken from Schunk [1983]; other collision frequencies and thermal conductivities were taken from Raitt et al. [1975].

Production and loss processes are similar to those in the one-stream version of the model. Ions are assumed to be produced only in the hemisphere of their origin, but can be lost at any point on the field line. $\mathrm{O}^{+}$ions are produced by photoionization and charge exchange of $\mathrm{O}$ with $\mathrm{H}^{+}$. $\mathrm{H}^{+}$ions are created by charge exchange of $\mathrm{H}$ with $\mathrm{O}^{+}$. $\mathrm{O}^{+}$is removed by recombination reactions with $\mathrm{N}_{2}$ and $\mathrm{O}_{2}$ and by charge exchange with $\mathrm{H} ; \mathrm{H}^{+}$is lost in charge exchange with $\mathrm{O}$. There is an additional production and loss mechanism for $\mathrm{H}^{+}$ions which is unique to the two-stream model: charge exchange of $\mathrm{H}^{+}$with $\mathrm{H}$. This will be a sink for the ions flowing out of the conjugate hemisphere and a source for the ions that originate in the given hemisphere. The following expression was used for the charge exchange frequency [cf. Banks and Kockarts, 1973, part A, p. 223]:

$$
v_{C E}\left(H^{+}, H\right)=1.0 \times 10^{-10} n(H) \sqrt{T\left(H^{+}\right)+T_{n}}
$$

The loss rate for the outflowing ions is given by the product of this frequency and the density of the outflowing ions; the production rate for the inflowing ions is equal to the loss rate for the outflowing ions.

The neutral atmosphere model used was MSIS-86 [Hedin, 1987] for June solstice during solar minimum conditions. A neutral wind profile was derived from the vector spherical harmonic (VSH) model [Killeen et al., 1987] and depends on the hemisphere. The solar zenith angle and $\mathrm{O}^{+}$photoproduction rate were found for each computational cell; these were calculated by using software taken from a conductivity model developed by Rasmussen et al. [1988]. The neutral atmosphere parameters, solar zenith angles, and photoproduction rates are updated every $15 \mathrm{~min}$ in order to simulate the rotation of the flux tube. Heating of thermal electrons by photoelectrons is included in the model; however, the work presented in this paper was done for nighttime conditions, so there was no electron heating except at high altitudes, where a minimum heating rate equal to $2.2 \times 10^{-13} \mathrm{erg} \mathrm{cm}^{-3} \mathrm{~s}^{-1}$ was imposed in order to match plasmaspheric temperature observations due to Comfort et al. [1985].

The model flux tube extends from $200-\mathrm{km}$ altitude in one ionosphere to $200-\mathrm{km}$ altitude in the conjugate ionosphere. Boundary conditions for the inflowing ions are set at $200-\mathbf{k m}$ altitude in the ionosphere of origin. Boundary conditions for the outflowing $\mathrm{H}^{+}$ions are set at about $400-\mathrm{km}$ altitude, whereas boundary conditions for the outflowing $\mathrm{O}^{+}$ions are set at about $1500 \mathrm{~km}$. The altitudes where these conditions are set were raised from $200 \mathrm{~km}$ to avoid numerical problems; however, it should be noted that this can result in reflective boundaries, due to the lower neutral densities at these higher altitudes. The boundary conditions for the inflowing ions are that the ions be in chemical and thermal equilibrium with the neutrals and that the field-aligned velocity of the ions is zero. The inflowing $\mathrm{O}^{+}$boundary density is found by using photochemical equilibrium during the day but is set to $1.5 \times 10^{3} \mathrm{~cm}^{-3}$ at night. The inflowing $\mathrm{H}^{+}$boundary density is found by assuming charge exchange equilibrium with $\mathrm{O}^{+}$. The boundary densities for the outflowing ions are found by extrapolating from interior points. For the outflowing $\mathrm{H}^{+}$ions the boundary temperatures are found by extrapolating from interior points, but for the outflowing $\mathrm{O}^{+}$ions the boundary temperature is set to the temperature in the cell adjacent to the boundary. The boundary velocities are set to the velocities in the cell adjacent to the boundary if these velocities are downward; otherwise they are set to zero.

The equations are solved by using a combined Godunov scheme/Crank-Nicholson method with dimensional splitting, as described by Guiter and Gombosi [1990]. The cell size was $-12.6 \mathrm{~km}$. The model was run in two time steps: a small time step at low altitudes and a larger time step at high altitudes, the division between low and high altitudes being about $1500 \mathrm{~km}$. The low-altitude time step was $6.25 \times 10^{-3} \mathrm{~s}$; the ratio of the high- and low-altitude time steps varied between 1 and 5 . At times, however, it was necessary to use a time step as low as $3.125 \times 10^{-3} \mathrm{~s}$ for all points on the field line. In addition, to prevent numerical problems caused by $\mathrm{O}^{+}$shocks at high altitudes, an artificially high (0.1) momentum transfer collision frequency for $\mathrm{O}^{+}$ions with neutrals was used when the ratio of the number density of $\mathrm{O}^{+}$to that of electrons was less than 0.01 ; these $\mathrm{O}^{+}$shocks were also seen in steady state results found with the one-stream version of the model, and were not due to refilling.

\section{Results}

The initial condition was chosen to simulate a density depletion. To do this, the initial $\mathrm{H}^{+}$density profile above 2000 $\mathrm{km}$ altitude was determined by the following formula:

$$
n(\lambda)=n\left(\lambda_{0}\right)\left(\frac{\sin \lambda}{\sin \lambda_{0}}\right)^{4}
$$

where $\lambda$ is magnetic latitude and $\lambda_{0}$ is the magnetic latitude that corresponds to $2000-\mathrm{km}$ altitude; a minimum density was also imposed, equal to $10^{-3}$ times the density of the other $\mathrm{H}^{+}$stream in the first cell in from the boundary. This is similar to that used by Rasmussen and Schunk [1988]. The initial density profiles for all species are shown in Figure 1 as a function of magnetic latitude. Diurnally reproducible results for June solstice conditions at midnight on an $L=4$ field line from a one-stream version of the model were used to determine the $\mathrm{O}^{+}$density everywhere and the $\mathrm{H}^{+}$density up to $2000-\mathrm{km}$ altitude for each stream in the hemisphere of origin. The $\mathrm{O}^{+}$density profiles are not necessarily realistic at high altitudes because of the artificial resistance used in the one-stream model. The electron densities were determined by using quasi-neutrality. The initial temperature profiles were also taken from the one-stream results. The initial velocities were assumed to be zero.

Because of the density depletion, the ion gases expand upward into the low-density regions. This can be seen in Figure 2, which shows velocity and density profiles for the southern stream of $\mathrm{H}^{+}$ions between 0 and $15 \mathrm{~min}$. It should be noted that positive velocities are upward in the northern hemisphere but downward in the southern hemisphere, and that positive latitudes correspond to the northern hemisphere. The velocities are highly supersonic with respect to the ion-acoustic speed $C_{s}$, where the ion-acoustic speed is defined by

$$
C_{s}=\sqrt{\frac{k}{m_{i}} T_{e}}
$$




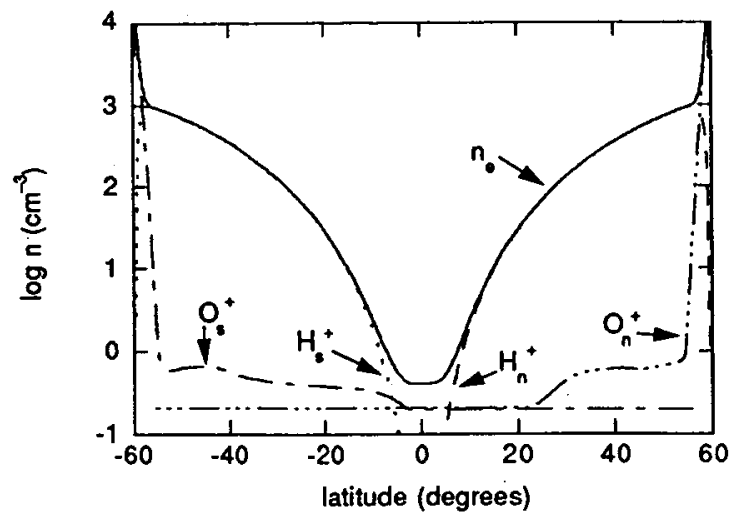

Figure 1. Initial density profiles as a function of magnetic latitude used in the two-stream calculation on an $L=4$ field line. The solid line denotes electrons, the dotted line shows the $\mathrm{H}^{+}$southern stream, the dashed line shows the $\mathrm{H}^{+}$northern stream, the dot-dashed line corresponds to the $\mathrm{O}^{+}$southern stream, and the dash-dot-dot-dot line corresponds to the $\mathrm{O}^{+}$ northern stream.

At 5 min the ion-acoustic Mach number is approximately 3.2. As shown by Singh [1988], if the ion-acoustic Mach number is greater than 2.3, counterstreaming is expected to occur. The stream has crossed the equator by $5 \mathrm{~min}$ and by $15 \mathrm{~min}$ the density front has began to steepel.. In the northern hemisphere the velocity changes largely as a result of advection of the velocity fronts. It can also be seen that there is upward flow in both the southern and the northern hemispheres. The upward flow in the northern hemisphere is a consequence of the initial conditions, which specify a low initial density of southern

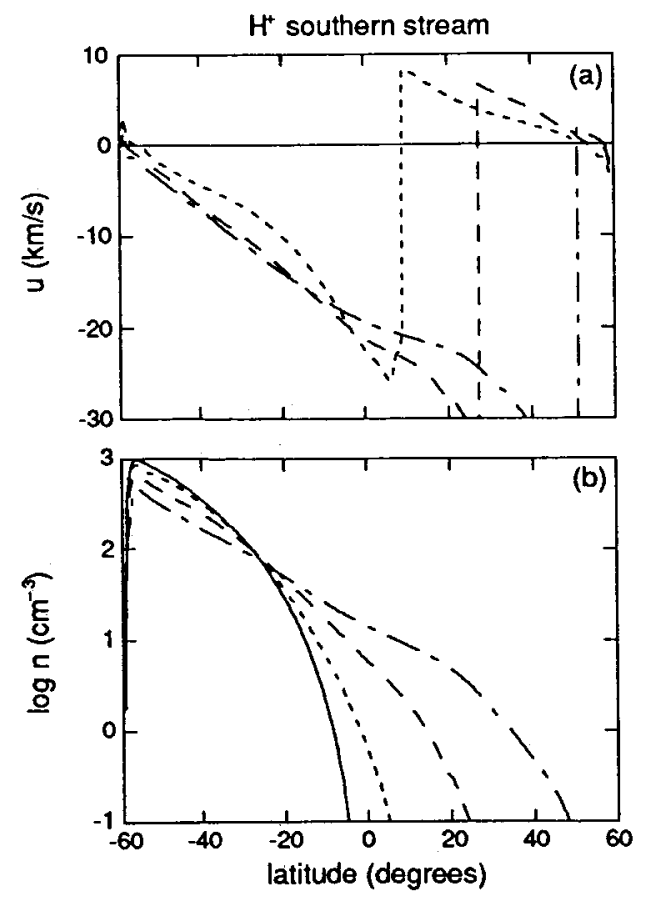

Figure 2. (a) Velocity and (b) density profiles as a function of magnetic latitude for the $\mathrm{H}^{+}$southern stream. The solid line shows the initial profiles, the dotted line gives results after 5 $\mathrm{min}$, the dashed line gives results after $10 \mathrm{~min}$, and the dotdashed line shows results at $15 \mathrm{~min}$. stream ions in the northem hemisphere; these ions flow upward in response to the upward electric field. The density front passes into the northern hemisphere practically unimpeded. Similar results were found for the stream from the northern hemisphere, with the density front in that case passing from the northern into the southern hemisphere. The electron density fills in rapidly; by $15 \mathrm{~min}$ the equatorial electron density is approximately $30 \mathrm{~cm}^{-3}$.

It can also be seen that in this initial period, downward flow develops at low altitudes $(-400 \mathrm{~km})$. This is a consequence of the initial density distribution below $2000-\mathrm{km}$ altitude, which corresponds to that for quiet-time nighttime conditions; for such conditions, downward flow develops.

By $17.5 \mathrm{~min}$ the density front has passed through the outflow boundary. Thereafter the density builds up near the boundary owing to the large downward fluxes. This can be seen in Figure 3, which shows velocity and density profiles at 20 and $25 \mathrm{~min}$ for the southern stream of $\mathrm{H}^{+}$ions. There is also an upward velocity spike at approximately $860 \mathrm{~km}$ in the southern hemisphere which is accompanied by a local density depletion; this develops into a reverse shock, which moves upward (a reverse shock is one where the velocity changes from a high to low magnitude while the density changes from a low to high value). The maximum velocity in this spike is close to the sound velocity.

The development of the velocity spike is shown in Figure 4, which shows velocity and density profiles in the southern hemisphere between 12.5 and $17.5 \mathrm{~min}$ for the southern stream of $\mathrm{H}^{+}$ions; it should be recalled that in this region negative velocities are upward. The velocity spike begins to develop as early as $12.5 \mathrm{~min}$. The density profile at $17.5 \mathrm{~min}$ shows the beginning of the density depletion. From this figure it can also be seen that in this time period the upward velocity
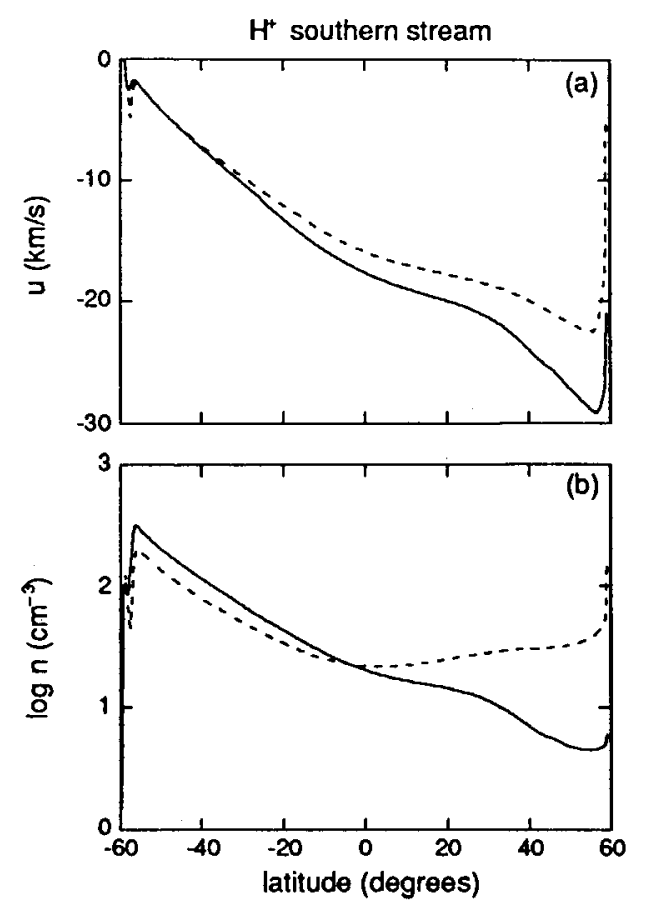

Figure 3. (a) Velocity and (b) density profiles as a function of magnetic latitude for the $\mathrm{H}^{+}$southern stream. The solid line shows results at $20 \mathrm{~min}$, and the dotted line gives results at 25 $\min$. 

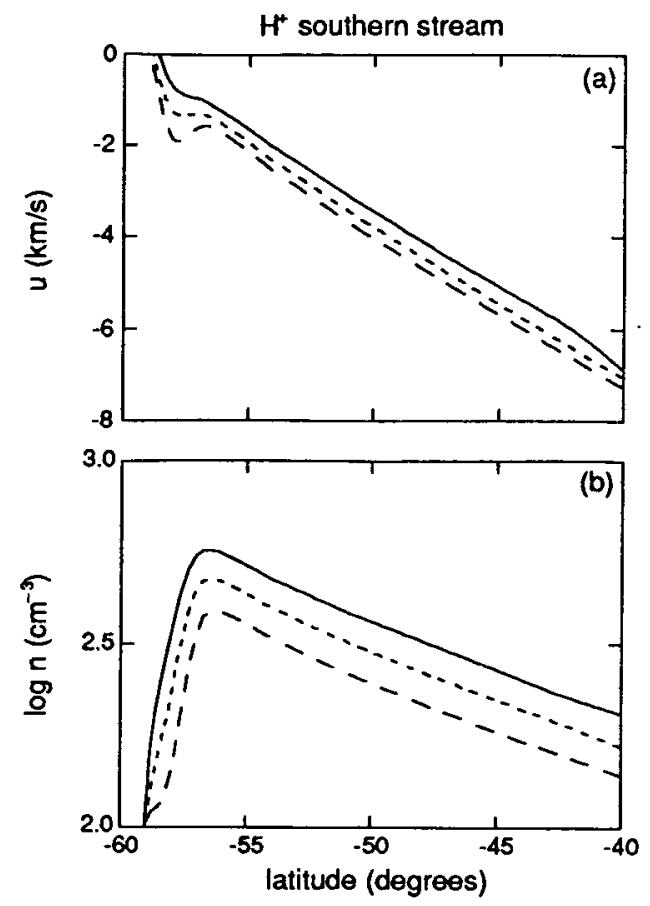

Figure 4. (a) Velocity and (b) density profiles as a function of magnetic latitude for the $\mathrm{H}^{+}$southern stream, between -60 and -40 degrees. The solid line shows results at $12.5 \mathrm{~min}$, the dotted line gives results at $15 \mathrm{~min}$, and the dashed line gives results at $17.5 \mathrm{~min}$.

throughout this region increases, driven by the upward electric field and by the density gradient force at high enough altitudes. Figure 5 shows altitude profiles of electron and ion (southern streams) density at $\mathbf{1 2 . 5} \mathrm{min}$ in the southern hemisphere. The velocity spike develops in the transition region where $\mathrm{O}^{+}$is the major ion and the $\mathrm{H}^{+}$density increases with altitude, which means that the density gradient force is downward and the momentum transfer collision frequency of $\mathrm{H}^{+}$with $\mathrm{O}^{+}$ions is relatively small. This is also the region where the electron pressure gradient changes drastically with altitude, resulting in the rapid decrease of the electric field with increasing altitude. In

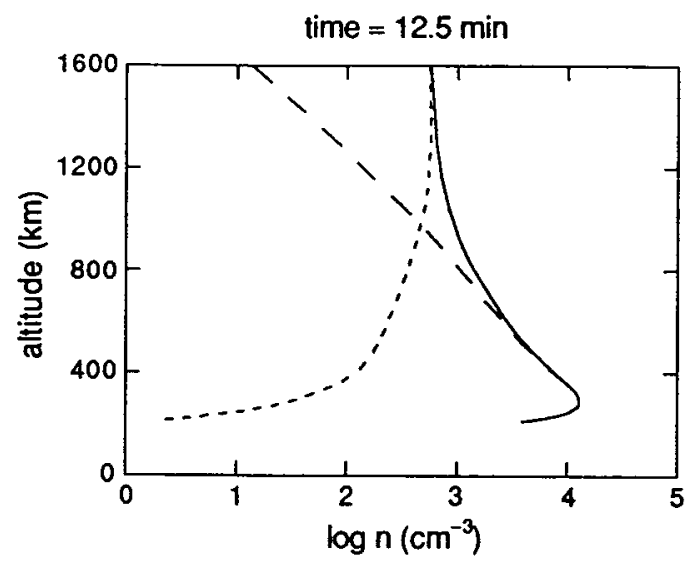

Figure 5. Altitude profiles of electron and ion (southern streams) density at $12.5 \mathrm{~min}$ in the southern hemisphere. The solid line refers to electron density, the dotted line refers to $\mathrm{H}^{+}$ density, and the dashed line refers to $\mathrm{O}^{+}$density. this region the most important forces (for the southern stream of $\mathrm{H}^{+}$ions) are the electric, density gradient, and gravitational forces; the sum of these forces is upward and significantly larger at about $860-\mathrm{km}$ altitude than at higher altitudes. This can be seen in Figure 6, which shows altitude profiles of the electric, density gradient, and total forces (for the southern stream of $\mathrm{H}^{+}$ions) in the southern hemisphere between 15 and $20 \mathrm{~min}$; it should be noted that positive forces are downward in this region and that the force scale for the total force profile is different from that for the other two profiles. As a result, the velocity increases more at about $860-\mathrm{km}$ altitude. The velocity rise causes more flux to leave this region than the ionosphere supplies, resulting in a local density depletion. This in turn causes the electron density to decrease, which causes the electric field to increase as the electron pressure gradient does not
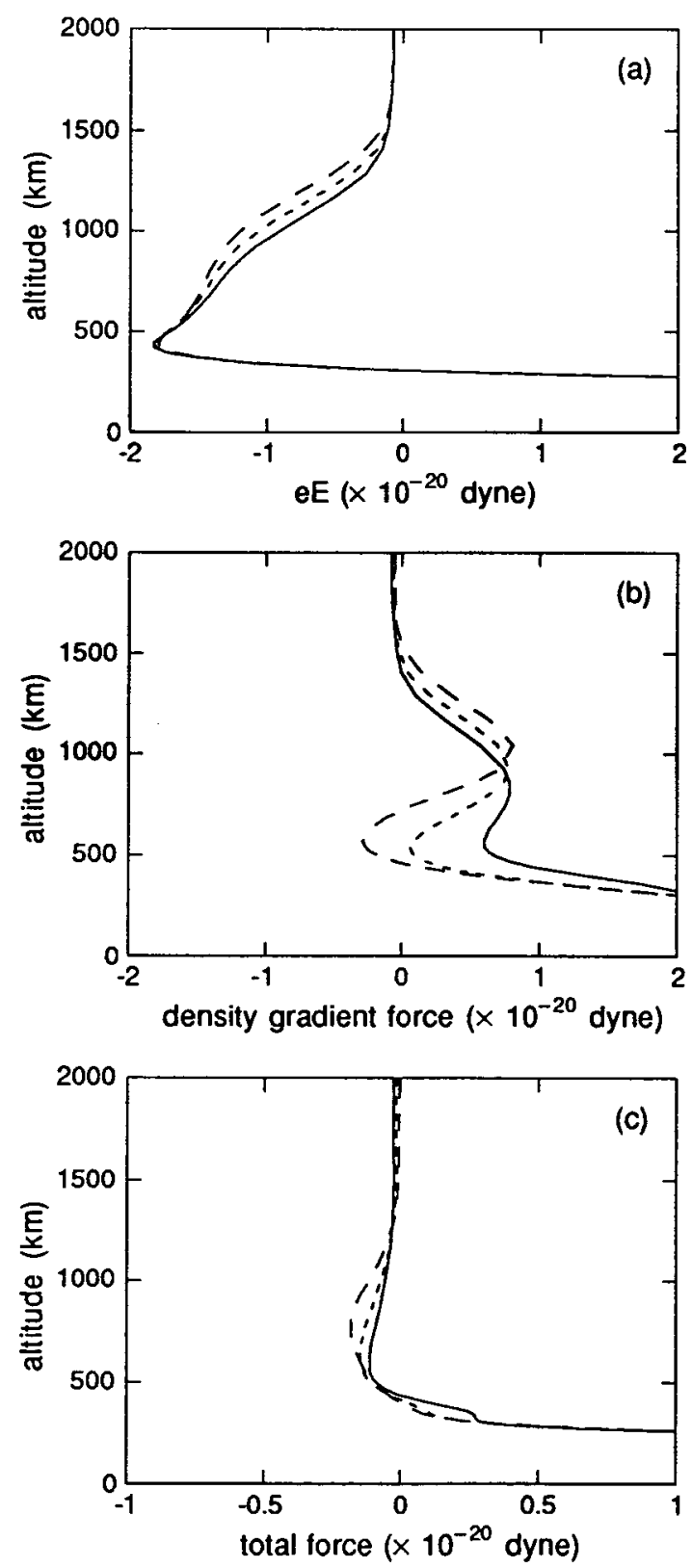

Figure 6. Altitude profiles of the (a) electric, (b) density gradient, and (c) total force in the southern hemisphere. The solid line shows results at $15 \mathrm{~min}$, the dotted line gives results at $17.5 \mathrm{~min}$, and the dashed line gives results at $20 \mathrm{~min}$. 
change significantly. From Figure 6 it can be seen that the increase of the electric field with time is partially offset by the increase in the downward density gradient force, so that the total force increases in only a limited altitude range. The result is that the velocity rises more in that altitude range, leading to the velocity spike.

Figure 7 shows altitude profiles of electron and ion (southern streams) density at $20 \mathrm{~min}$ in the southern hemisphere. At this time the density profile for the southern stream of $\mathrm{H}^{+}$ions has a broad minimum centered at about $740-\mathrm{km}$ altitude. This profile is similar to that found by Raitt et al. [1975] when there is strong upward flow out of the ionosphere, as there is in this case. This density structure steepens into a shock, which then moves upward.

By 30 min a shock has developed near the northern boundary and then moves upward. This can be seen in Figure 8, which shows velocity and density profiles at 30 and $40 \mathrm{~min}$ for the southem stream of $\mathrm{H}^{+}$ions. This develops as the velocity is slowed down (starting at about $20 \mathrm{~min}$ ) in the northern hemisphere in the 400 - to $500-\mathrm{km}$ altitude range, first by friction with neutrals and then by the density gradient force (the density increases toward the boundary). The velocity is slowed down from a supersonic value, which means that a shock must form. By 40 min the shock strength has diminished somewhat, although it continues to move upward. Also, by $40 \mathrm{~min}$ the reverse shock in the southern hemisphere has developed into a forward shock, which propagates with a shock in the northern stream of $\mathrm{H}^{+}$ions. Figure 9 shows density results in the southern hemisphere for electrons and both $\mathrm{H}^{+}$fluids, at 40 and 45 min; it can be seen that the shocks in the two $\mathrm{H}^{+}$fluids are moving together. The motions of these two shocks are coupled by the polarization electric field, which at this time is generated by the northern stream of $\mathrm{H}^{+}$ions.

Figure 10 shows velocity and density results between 50 and $60 \mathrm{~min}$ for the southern stream of $\mathrm{H}^{+}$ions. The shock that formed near the northern boundary continues to move upward and is slowly dissipating; by $60 \mathrm{~min}$ it has almost reached 20 degrees latitude. Also, at $50 \mathrm{~min}$ there is upward flow in the northern hemisphere of $\mathrm{H}^{+}$ions which come from the southern hemisphere. This flow is driven by both the density gradient force and the electric field (which is directed toward the equator). The shock that originated in the southern hemisphere

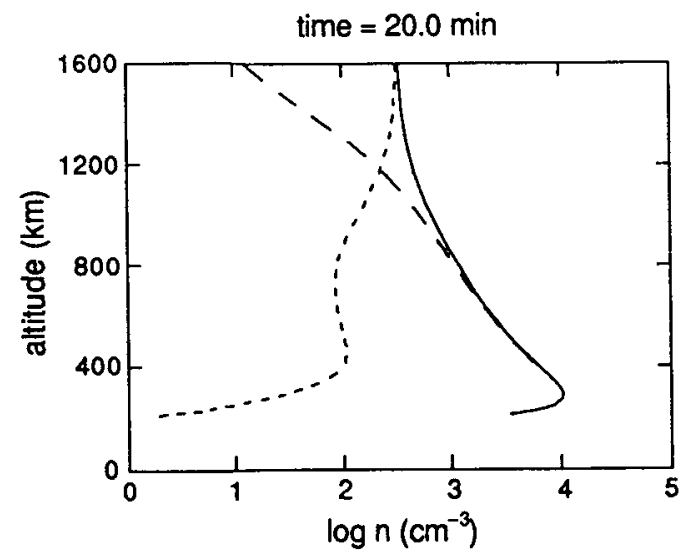

Figure 7. Altitude profiles of electron and ion (southern streams) density at $20 \mathrm{~min}$ in the southern hemisphere. The solid line refers to electron density, the dotted line refers to $\mathrm{H}^{+}$ density, and the dashed line refers to $\mathrm{O}^{+}$density.

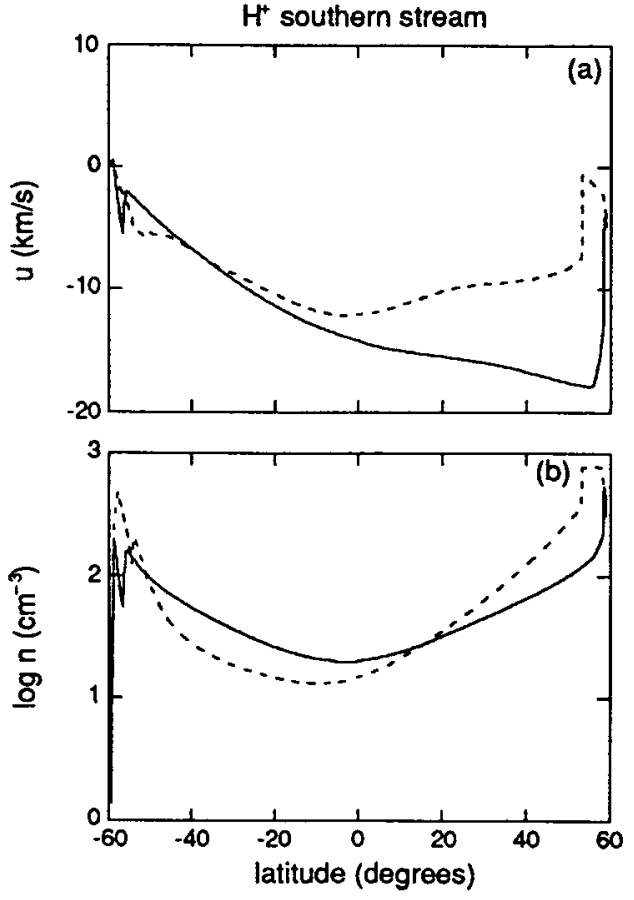

Figure 8. (a) Velocity and (b) density profiles as a function of magnetic latitude for the $\mathrm{H}^{+}$southern stream. The solid line shows results at $\mathbf{3 0} \mathrm{min}$, and the dotted line gives results at $\mathbf{4 0}$ $\min$.

also moves upward. What is especially interesting, however, is that a shock develops near the equator. The steepening begins at $55 \mathrm{~min}$ and is almost complete at $60 \mathrm{~min}$. This shock forms as supersonic flow from the southern end is slowed down in the northern hemisphere by pressure gradients as well as the
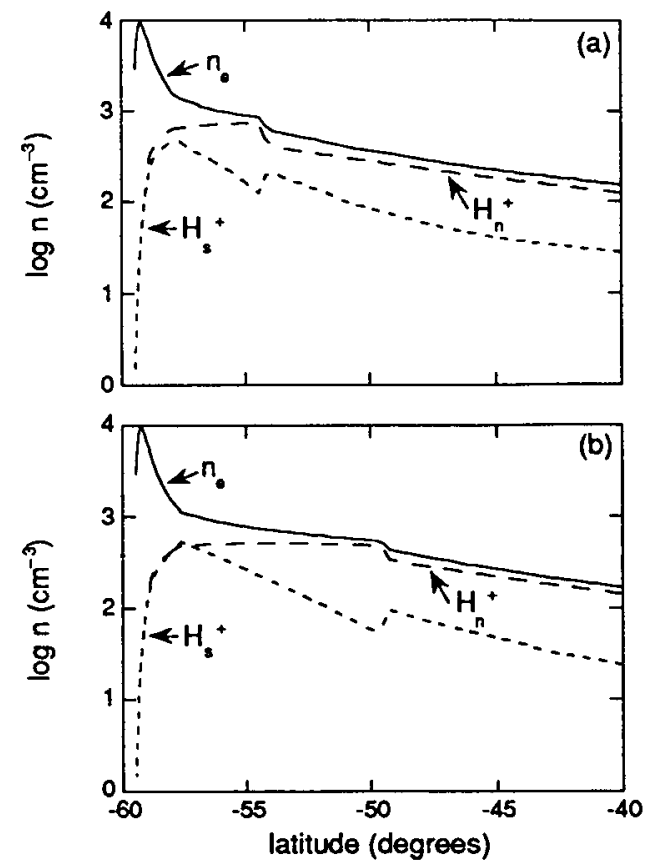

Figure 9. Density profiles as a function of magnetic latitude (between -60 and -40 degrees) at (a) $40 \mathrm{~min}$ and (b) $45 \mathrm{~min}$. The solid line denotes electrons, the dotted line shows the $\mathrm{H}^{+}$ southern stream, and the dashed line shows the $\mathrm{H}^{+}$northern stream. 

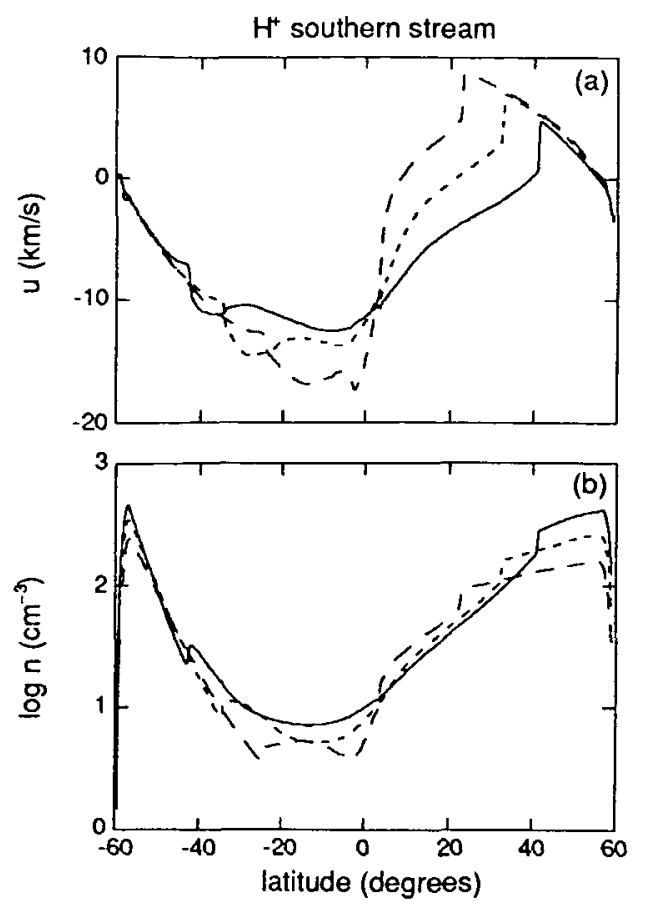

Figure 10. (a) Velocity and (b) density profiles as a function of magnetic latitude for the $\mathrm{H}^{+}$southem stream. The solid line shows results at $50 \mathrm{~min}$, the dotted line gives results at 55 $\mathrm{min}$, and the dashed line gives results at $60 \mathrm{~min}$.

electric field. In addition, the flow is accelerated in the southern hemisphere (between -20 and 0 degrees latitude), primarily by the electric field. This shock subsequently moves toward the southern end. A similar shock develops in the stream which originates in the northern hemisphere. As these shocks develop, the electron density between them decreases and continues to decrease as they move toward each other.

The possibility exists that these shocks are artificial, especially because in each stream there are large upward flows in both hemispheres when they form. Furthermore, when an attempt was made to redo the portion of the run from 45 to 60 min with the cell size equal to about $6.3 \mathrm{~km}$ (half the original size) and the same Courant number, it was found that very narrow, spiky shock structures had developed by $55 \mathrm{~min}$, in the approximate place where the large shock formed; when the high-altitude time step was reduced, the results were similar to those found with the larger cell size. It should be recalled that with the larger cell size the large shocks formed at about 60 min and were not spiky. This enhances the possibility that these shocks are numerical artifacts, related to cell size or time step problems. Because these shocks are probably artificial, further results will not be discussed.

\section{Summary}

We have modeled plasmaspheric refilling following a density depletion on an $L=4$ field line, using a two-stream version of the plasmaspheric model we developed. The initial density profile was chosen to be similar to that of Rasmussen and Schunk [1988]. Initially, the upwelling streams pass through each other without being impeded. Electrostatic shocks are not set up when the streams meet at the equator because the stream velocities are highly supersonic with respect to the ion-acoustic speed. In addition, Singh [1990] has noted that electrostatic shocks are not likely to be set up in hydrodynamic models with grid spacings of the order of tens of kilometers, since the scale length for the relevant plasma processes is of the order of a few Debye lengths $(\sim 10 \mathrm{~m})$. It should also be noted that perpendicular heating caused by wave-particle interactions in the equatorial region is not included. Such heating has been shown [Singh and Torr, 1990; Singh and Chan, 1992] to slow down the ion streams enough to inhibit the interhemispheric plasma exchange. After the stream reaches the conjugate ionosphere, a shock develops there which then moves upward. This shock slowly dissipates. A reverse shock also forms in each stream in the hemisphere of origin and moves up; however, the development of the reverse shocks could depend on the initial conditions used. After 1 hour, large shocks develop in each stream near the equator. These shocks then move back toward the equator and downward. However, these shocks are probably artificial, because counterstreaming flows occur in each $\mathrm{H}^{+}$fluid, which the model can only handle by creating shocks.

While the results are similar to those of Rasmussen and Schunk [1988], there are differences. Their model is similar to our model; however, the ion and electron energy equations are included in our model, and our model flux tube extends much lower in the ionosphere, to altitudes where $\mathrm{O}^{+}$(which is included in our model) is the dominant ion species. The large shocks that Rasmussen and Schunk [1988] find are a result of a reverse shock which develops in the northern hemisphere, moves past the equator, and then reverses direction. Such reverse shocks also develop in our results, but the large shocks develop independently of them. After the large shocks form, they cross the equator and continue to move downward. However, the large shocks that develop in our results are probably artificial.

A similar study was done by Wilson et al. [1992], who used a semikinetic model that included Coulomb collisions for ionion interactions. In this model, each simulation particle represents many actual particles; the simulation particles are acted on by gravitational, magnetic mirror, and electric field forces. The electric field is found by using a Boltzmann relation between electron number density and electric potential. In the model the bases of the flux tube are at about 1900-km altitude. At each base, ions are injected into the flux tube, with a velocity distribution that corresponds to the upgoing half of a nondrifting Maxwellian. Plasmaspheric refilling was studied on a variety of $L$ shells, including $L=4$. In each case the same sequence of events occurs. Initially, a polar wind type outflow from each ionosphere is present. Within an hour, two counterstreaming beams exist along the entire flux tube. The phase space gap between these two beams is filled in by collisional diffusion; when it has filled, the plasma is nearly isotropic and Maxwellian everywhere. What is most important, in view of the results presented in this paper, is that shock fronts are never produced, at the equator or any other point. Field-aligned structures in the equatorial region from $L=3$ to 5 have, however, been inferred from DE 1 observations, especially in the electron density and the $\mathrm{H}^{+}$density, temperature, and pitch angle distribution [Olsen et al., 1987; Olsen, 1992].

Limitations of our model include the restriction to isotropic temperatures and the inability to distinguish counterstreaming flows within one fluid. In some sense it is inconsistent to assume temperature isotropy when using a two-stream model, because if the collision frequencies are low enough so that the 
upwelling streams would not thermalize when they meet, then they are low enough for temperature anisotropies to be present. The inability to distinguish counterstreaming flows within one fluid is a problem if there is a large return flux that is about equal in magnitude to the flux flowing into the conjugate hemisphere, since then an artificial shock could be formed. Such return fluxes occur after the density of an ion stream rises in the conjugate hemisphere and the density gradient force drives the ions back. The large shocks that form near the equator are probably artificial, although this may be due to cell size or time step problems rather than the reasons given above. However, despite these limitations we have shown that initially the two $\mathrm{H}^{+}$streams interpenetrate, and that interesting shock structures develop in the ionospheres and propagate upward.

Acknowledgments. This work was supported by NASA grant NAGW1619 and by NSF grant ATM-9116858. One of us (S.M.G.) was supported by NASA Marshall Space Flight Center through the graduate student training grant NGT-50368 for part of this work. Acknowledgment is also made to the National Center for Atmospheric Research, sponsored by the National Science Foundation, for the computing time used in this research.

The editor thanks M. Schulz and S. Watanabe for their assistance in evaluating this paper.

\section{References}

Banks, P. M., and G. Kockarts, Aeronomy, Academic, San Diego, Calif., 1973.

Banks, P. M., A. F. Nagy, and W. 1. Axford, Dynamical behavior of thermal protons in the mid-latitude ionosphere and magnetosphere, Planet. Space Sci., 19, 1053, 1971.

Burgers, J. M., Flow Equations for Composite Gases, Academic, San Diego, Calif., 1969.

Comfort, R. H., J. H. Waite Jr., and C. R. Chappell, Thermal ion temperatures from the retarding ion mass spectrometer on DE $1, J$. Geophys. Res., 90, 3475, 1985.

Guiter, S. M., and T. I. Gombosi, The role of high-speed plasma flows in plasmaspheric refilling, J. Geophys. Res., 95, 10,427, 1990.

Guiter, S. M., T. I. Gombosi, and C. E. Rasmussen, Diurnal variations on a plasmaspheric flux tube: Light ion flows and $F$ region temperature enhancements, Geophys. Res. Lett., 18, 813, 1991.

Hedin, A. E., MSIS-86 thermospheric model, J. Geophys. Res., 92, 4649, 1987.

Killeen, T. L., R. G. Roble, and N. W. Spencer, A computer model of global thermospheric winds and temperatures, Adv. Space Res., 7(10), 207, 1987.

Olsen, R. C., Equatorially trapped plasma populations, J. Geophys. Res., $86,11,235,1981$.

Olsen, R. C., The density minimum at the Earth's magnetic equator, $J$. Geophys. Res., 97, 1135, 1992.
Olsen, R. C., S. D. Shawhan, D. L. Gallagher, J. L. Green, C. R. Chappell, and R. R. Anderson, Plasma observations at the Earth's magnetic equator, J. Geophys. Res., 92, 2385, 1987.

Raitt, W. J., R. W. Schunk, and P. M. Banks, A comparison of the temperature and density structure in the high and low speed thermal proton flows, Planet. Space Sci., 23, 1103, 1975.

Rasmussen, C. E., and R. W. Schunk, Multistream hydrodynamic modeling of interhemispheric plasma flow, J. Geophys. Res., 93, 14,557, 1988.

Rasmussen, C. E., R. W. Schunk, and V. B. Wickwar, A photochemical equilibrium model for ionospheric conductivity, J. Geophys. Res., 93, 9831, 1988.

Schulz, M., and H. C. Koons, Thermalization of colliding ion streams beyond the plasmapause, J. Geophys, Res., 77, 248, 1972.

Schunk, R. W., Mathematical structure of transport equations for multispecies flows, Rev. Geophys., 15, 429, 1977.

Schunk, R. W., The terrestrial ionosphere, in Solar-Terrestrial Physics, edited by R. L. Carovillano and J. M. Forbes, p. 609, D. Reidel, Norwell, Mass., 1983.

Singh, N., Refilling of a plasmaspheric flux tube: Microscopic plasma processes, in Modeling Magnetospheric Plasma, Geophys. Monogr. Ser., vol. 44, edited by T. E. Moore and J. H. Waite Jr., p. 87, AGU, Washington, D.C., 1988.

Singh, N., Comment on "Multistream hydrodynamic modeling of interhemispheric plasma flow" by C. E. Rasmussen and R. W. Schunk, $J$. Geophys. Res., 95, 17,273, 1990.

Singh, N., Role of ion temperature anisotropy in multistage refilling of the outer plasmasphere, Geophys. Res. Lett., 18, 817, 1991.

Singh, N., and C. B. Chan, Effects of equatorially trapped ions on refilling of the plasmasphere, J. Geophys. Res., 97, 1167, 1992.

Singh, N., and D. G. Torr, Effects of ion temperature anisotropy on the interhemispheric plasma transport during plasmaspheric refilling, Geophys. Res. Lett., 17, 925, 1990.

Singh, N., R. W. Schunk, and H. Thiemann, Temporal features of the refilling of a plasmaspheric flux tube, J. Geophys. Res., 91, 13,433, 1986.

Tanenbaum, B. S., Plasma Physics, McGraw-Hill, New York, 1967.

Wilson, G. R., J. L. Horwitz, and J. Lin, A semikinetic model for early stage plasmasphere refilling, 1 , Effects of Coulomb collisions, $J$. Geophys. Res., 97, 1109, 1992.

T.I. Gombosi and C.E. Rasmussen, Space Physics Research Laboratory, University of Michigan, Ann Arbor, MI 48109. (e-mail: tamas@spric.spri.umich.edu; rasmussn@jupiter.eecs.umich.edu)

S.M. Guiter, NASA/MSFC ES83, Space Sciences Laboratory, Huntsville, AL 35812. (e-mail: guiter@mpb.msfc.nasa.gov)

(Received March 24, 1993; revised November 7, 1994; accepted November 30, 1994.) 